\title{
Antibakterielle Inhaltsstoffe in Algen
}

\author{
1. Mitteilung \\ K.-W. GlombitZA \\ Pharmakognostisches Institut der Universität Bonn, Bonn
}

\begin{abstract}
Antibacterial components in algae. 1. Contribution. Antibiotic components and intensity activities have been determined in several seaweed species. In order to obtain suitable preparations revealing antibacterial activities, several methods have been tested with respect to stability and desiccating the material. Antibiotic properties have been determined in preparations of 9 Chlorophyceae, 16 Rhodophyceae, and 16 Phaeophyceae using as criterion populations growth of 4 gram-positive and 2 gram-negative bacteria strains. The most potent antibiotic properties occur in Chlorophyceae. Among the Rhodophyceae tested, only Chondrus crispus, Laurencia pinnatifida, Delesseria sanguinea, and - to a lesser degree - Rbodomela confervoides show considerable antibiotic activities. Among the Phaeophyceae, antibacterial potentials occur in Halidrys siliquosa, Fucus, Laminaria digitata, and Desmarestia viridis. The antibiotic compounds of Rhodophyceae and Chlorophyceae are mainly hydrophilic, whereas those of Phaeophyceae are in part extractible with hydrophobic solvents. Dumontia incrassata contains volatile compounds with a low antibiotic activity. The antibacterial properties of Dumontia incrassata are due to acidification of the culture medium by sulfuric acid, diffusing from the thalli into the agar.
\end{abstract}

\section{EINLEITUNG}

Bei der Suche nach antibakteriell wirksamen Substanzen wurden bisher überwiegend Pilze und Bakterien und erst später in steigendem Maße Spermatophyten untersucht. Ausgesprochen dürftig sind jedoch unsere Kenntnisse über antimikrobielle Eigenschaften und Stoffe bei Algen. Nach den bisher veröffentlichten Arbeiten wurden erst 131 Algenarten auf antibakterielle, 42 auf antifungale, 46 auf algostatische und 51 Arten auf antivirale Eigenschaften geprüft.

In vielen Fällen konnte keine Hemmwirkung nachgewiesen werden; in zahlreichen Fällen widersprechen sich die Ergebnisse der einzelnen Untersucher. Das ist nicht weiter verwunderlich, denn manchmal wurden zermörserte Thallusstücke (GUPTA \& SHRIvastava 1963, MCCUtCHEON et al. 1949, WELCH 1962), manchmal Atherextrakte aus mit Heißluft getrockneten Algen (Chesters \& Stott 1955, Roos 1957) und dann wieder methanolische oder wäßrige Auszüge aus eingefrorenen (AllEN \& DAwson 1960, MautNer et al. 1953, Olesen et al. 1964) oder getrockneten Algen (Roos 1957) in jeweils unterschiedlicher Konzentration geprüft. Vor einer genaueren Untersuchung einzelner ausgewählter Arten mußten deshalb in einem systematischen Programm ver- 
schiedene Algen auf unterschiedliche Weise aufgearbeitet und die verschiedenen $\mathrm{Zu}$ bereitungen auf ihre Wirksamkeit geprüft werden.

\section{MATERIAL UND METHODEN}

A lge n m a teria 1. Die Algen wurden 1964, 1965 und 1966 jeweils in den Monaten Mai-Juni in Helgoland oder 1967 im März an der Normandieküste an ihren natürlichen Standorten gesammelt und im Meerwasserbecken von Verunreinigungen befreit, ausgepreßt und kurz in Süßwasser nachgewaschen. Je nach dem Bestimmungs$\mathrm{z}$ weck wurden die Thatli entweder (a) mit der gleichen Volumen/Gewichtsmenge Alkohol übergossen und nach einer Vormahlung im Mix-Gerät zerkleinert, oder (b) möglichst rasch bei $-18^{0}$ eingefroren und bei dieser Temperatur aufbewahrt und transportiert, oder (c) die gefrorenen Thalli gefriergetrocknet, oder (d) in gefrorenem Zustand unter Zusatz von Trockeneis in der IKA-Analysenmühle oder einem Mix-Gerät gepulvert, oder (e) in einem Trockenschrank mit Frischluftgebläse entweder bei Raumtemperatur (Kaltluft) oder bei $60^{\circ}$ (Heißluft) getrocknet.

Frische oder getrocknete Thallusstücke und Pulver wurden mit einer mit Phosphatpuffer pH 6,5 angesetzten 2\%igen Lösung von Adulsion MH 300 mit $10 \%$ Athanol auf $120 \%$ des Frischgewichtes aufgefüllt und dispergiert. Die Extrakte wurden auf $20 \%$ des Frischgewichtes der extrahierten Alge aufgefüllt.

B a k te rienstä m e. Als Teststämme dienten Bacillus subtilis ATCC 6633, Bacillus cereus ATCC 9643, Staphylococcus aureus SG 511, Sarcina lutea ATCC 9341 sowie Escherichia coli und Protens vulgaris.

Agar-Lochplattentest. Die auf Schrägagarröhrchen gehaltenen Bakterienkulturen wurden auf Difco Antibiotic-Medium III bis zum Ende der logarithmischen Wachstumsphase vorgezogen und nach Messung der optischen Dichte soviel davon mit $50^{\circ}$ warmen Difco Antibiotic Medium I gemischt, daß die Einsaat bei Escherichia coli, Staphylococcus aureus $1 \cdot 10^{6}$, bei Bacillus cereus $2,5 \cdot 10^{5}$, bei Protens vulgaris und Sarcina lutea $1 \cdot 10^{5} \mathrm{Keime} / \mathrm{ml}$ und bei der Sporensuspension von Bacillus subtilis 2,5 $10^{4}$ Sporen/ml betrug. Der beimptte Agar wird in einer Schichtdicke von 2,2 $\mathrm{mm}$ ausgegossen, und nach dem Erkalten wurden Löcher von $12 \mathrm{~mm} \phi$ ausgestochen, die mit 0,2 ml Prüflösung oder Suspension gefüllt wurden. Man bebrütet bei Escherichia coli, Bacillus subtilis und Bacillus cereus 6 bis 6\%/2, Staphylococcus aureus, Proteus vulgaris 15 bis 16 und Sarcina lutea 20 bis 24 Stunden bis zur Messung der Hemm-, Farb- und Förderungszonen.

\section{ERGEBNISSE}

Das auf unterschiedliche Weise aufgearbeitete Material wurde im Agar-Lochplattentest auf seine antibakteriellen Eigenschaften geprüf. Beim Vergleich der verschiedenen Aufarbeitungsmethoden ergab sich folgendes: (1) Das gefrorene und unmittelbar vor der mikrobiologischen Untersuchung aufgetaute Material hat in fast allen Fällen die stärkste Hemmwirkung. (2) Das gefriergetrocknete Material ist fast immer 
etwas weniger wirksam. Der Unterschied gegen (1) ist aber in den meisten Fällen minimal. Lediglich bei der sowieso nur gegen Bacillus subtilis schwach wirksamen Dumontia incrassata geht die Wirkung völlig verloren. (3) Das im Warmluftstrom getrocknete Material zeigt im allgemeinen einen sehr deutlichen Wirkungsabfall. Dieser ist gegenüber Bacillus subtilis bei Acrosiphonia centralis, Chorda filum, Codium fragile, Desmarestia aculeata, Enteromorpha intestinalis, Porphyra umbilicalis, Rhodomela confervoides, Laminaria saccharina und L. byperborea besonders groß; nur geringfügig ist er bei Delesseria sanguinea, Fucus vesiculosus, Polysiphonia urceolata, Scytosiphon lomentarius, Ulva lactuca und Laminaria digitata. Chondrus crispus, Dumontia incrassata, Fucus serratus und Halidrys siliquosa schließlich sind fast genauso oder stärker wirksam wie bei der Verwendung von eingefrorenem Material. (4) Auch bei den im Kaltluftstrom getrockneten Algen geht ein erheblicher Prozentsatz der Hemmstoffe verloren. (5) Bei all diesen Versuchen sind die Ansätze mit Adulsion-Phosphatpuffer auf $120 \%$ des Gewichtes der frischen Thallusstücke verdünnt worden. Algen mit nur schwacher Hemmwirkung oder mit langsam diffundierenden Stoffen können bei dieser Untersuchungsweise unbeachtet bleiben, und außerdem kann sich der Zerkleinerungsgrad sehr stark auf das Ergebnis auswirken. Deshalb wurden auch eingedickte alkoholische Extrakte geprüf. Die Extrakte wurden in soviel Adulsion aufgenommen, daß $1 \mathrm{ml}$ Prüflösung $5 \mathrm{~g}$ Frischmaterial entsprach. Algen, die auch unter diesen Bedingungen keine Hemmwirkung zeigen, können als unwirksam gelten.

Der Ubersichtlichkeit halber werden außer bei den Chlorophyceen (Tab. 1) in den Tabellen 2 und 3 nur die mit Thalluspulver und Extrakten gewonnenen Werte aufgeführt werden.

\section{Chlorophyceae}

Wie Tabelle 1 zeigt, sind alle Chlorophyceen gegen gram-positive und einige auch gegen gram-negative Bakterien wirksam. Monostroma undulatum und Cladophora rupestris sind innerhalb der Grünalgen die schwächsten Hemmstoffbildner. In Obereinstimmung damit haben auch alle anderen Untersucher immer eine Hemmung grampositiver Organismen festgestellt, wenn sich auch im antibakteriellen Spektrum und bei der Wirkungsintensität Unterschiede gezeigt haben. Eine besonders auffällige Differenz ergibt sich zu Roos (1957), deren Kaltwasserextrakte aus Enteromorpha, Ulva und Codium nie Staphylococcus aureus hemmten. Bei orientierenden Versuchen zur fraktionierten Extraktion der Hemmstoffe zeigte sich, daß die mit lipophilen Lösungsmitteln gewonnenen Extrakte meist nur eine geringe Hemmwirkung gegen Bacillus cereus haben, während methanolische oder wäßrige Auszüge stark wirksam sind.

\section{Rhodophyceae}

Im Gegensatz zu der durchweg starken Antibiose der Chlorophyceen ist das Bild bei den Rhodophyceen (Tab. 2) sehr uneinheitlich. B. subtilis wird zwar auch hier immer, wenn zum Teil auch nur sehr schwach oder nur von den Extrakten, gehemmt. Eine Beeinflussung gram-negativer Stämme ist nur selten und dann zumeist auch nur 


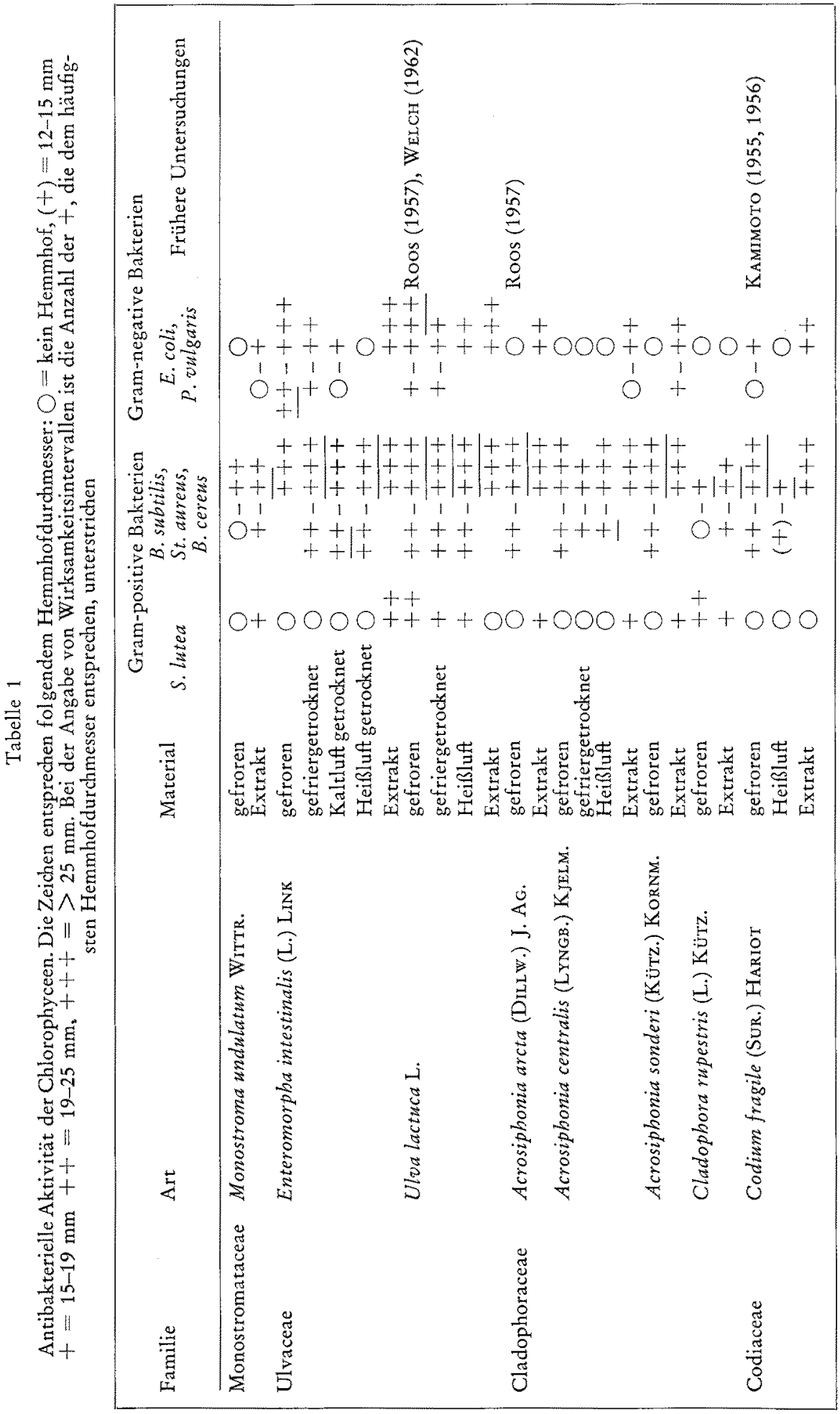


schwach festzustellen. Die Algen mit den stärksten Hemmstoffen sind Laurencia pinnatifida, Delesseria sangninea und Chondrus crispus sowie - schon deutlich abgeschwächt Plocamium vulgare. Die sowieso nur schwach wirksame Dumontia incrassata ist die einzige Alge, deren Hemmstoffe bei der Gefriertrocknung völlig verloren gehen. Es handelt sich dabei um wasserdampfflüchtige Stoffe, die wir auf Grund dieser Eigenschaften anreichern konnten.

Auffallenderweise schwanken die Hemmhofdurchmesser der verschiedenen Ernten von Rhodomela sehr stark. Das dürfte auf Grund der Befunde von Roos (1957) darauf zurückzuführen sein, daß diese Alge ihre antibakteriellen Eigenschaften zu Beginn des Frühjahrs weitgehend verliert, so daß wir nur 1964 aktives Material ernten konnten.

Während sich bei den Chlorophyceen fast nie ein Farbhof um das Einsaatloch in der Agarplatte zeigte, tritt dieser bei den Rhodophyceen außer bei Chondrus crispus in den Versuchen mit gefrorenem und getrocknetem Material immer, bei den Extrakten selten und nur sehr viel kleiner auf. Der wohl von Phycoerythrin hervorgerufene Farbhof steht in keinem Zusammenhang mit der Antibiose. Bei einer fraktionierten Extraktion von Chondrus crispus mit verschiedenen Lösungsmitteln in einer eluotropen Reihe werden durch die lipophilen Flüssigkeiten keine oder nur wenig Hemmstoffe herausgelöst. Die größten Anteile finden sich im Methanolextrakt. Auch hier scheinen also wie bei den Chlorophyceen relativ hydrophile Hemmstoffe vorzuliegen.

\section{Phaeophyceae}

Die Phaeophyceen sind höchstens in Form ihrer Extrakte gegen gram-negative Bakterien wirksam. Gegen gram-positive Organismen sind Chorda flum, Chordaria flagelliformis, Scytosiphon lomentarius, Desmarestia aculeata, Laminaria saccharina, Bifurcaria bifurcata, Himanthalia elongata und merkwürdigerweise auch Ascopbyllum nodosum durchweg von geringer und stark schwankender Aktivität (vgl. Tab. 3).

Mit Ascopbyllum nodosum haben andere Autoren z. T. sehr große Hemmhöfe erhalten (VACCA \& Walsh 1954). Die ausgesprochen niedrigen Werte in unseren Versuchen dürften wohl auf den frühen Erntezeitpunkt zurückzuführen sein, da Ascophyllum nodosum sein Wirkungsmaximum erst im Herbst erreicht (VACCA \& WatsH 1954). Die stärkste Antibiose zeigten in unseren Versuchen bei den Phaeophyceen Halidrys siliquosa und Fucus serratus neben Desmarestia viridis.

Einige Phaeophyceen (Pelvetia, Halidrys) geben Farbstoffe an den Agar ab. Die gelb bis gelbbraun gefärbten Zonen überschritten selten die Größe der Hemmhöfe. Sie scheinen mit den Hemmstoffen in Zusammenhang zu stehen.

Die Größe der Hemmhöfe ist stark von der Bebrütungsdauer abhängig. Bei den Chlorophyceen und bei einigen Rhodophyceen wachsen die Hemmhöfe bei einer langen Inkubationszeit von außen her allmählich zu, bei den Phaeophyceen dagegen werden sie meist größer, indem die Entwicklung bereits sichtbarer Kolonien im äußeren Bereich gestört wird und die Bakterien z. T. abgetötet werden. Die Hemmstoffe scheinen also nicht nur bakteriostatisch, sondern bakterizid zu sein und nur langsam zu diffundieren.

Die Frage nach dem Hemmstoff ron Desmarestia viridis ist einfach zu beantwor- 


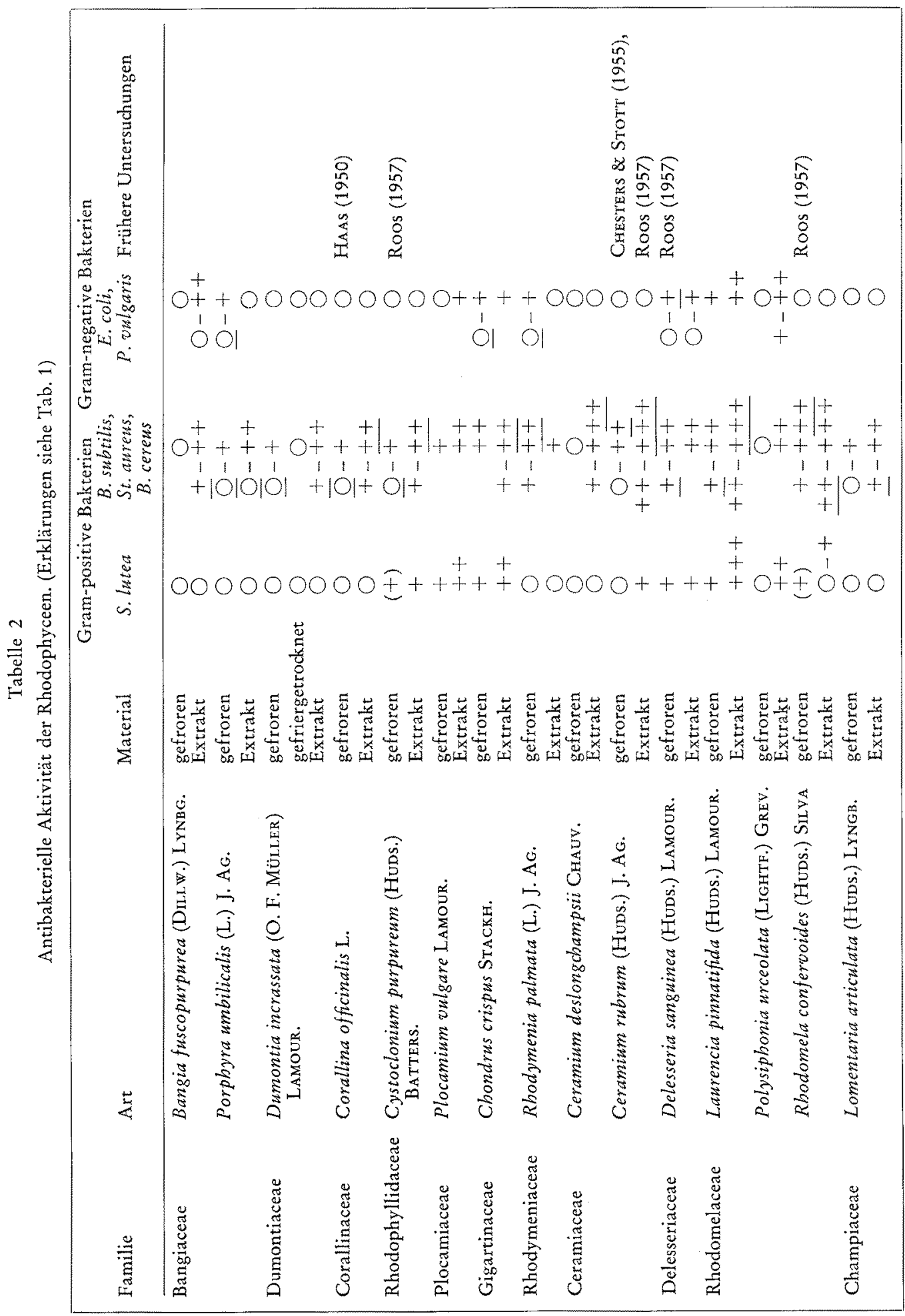




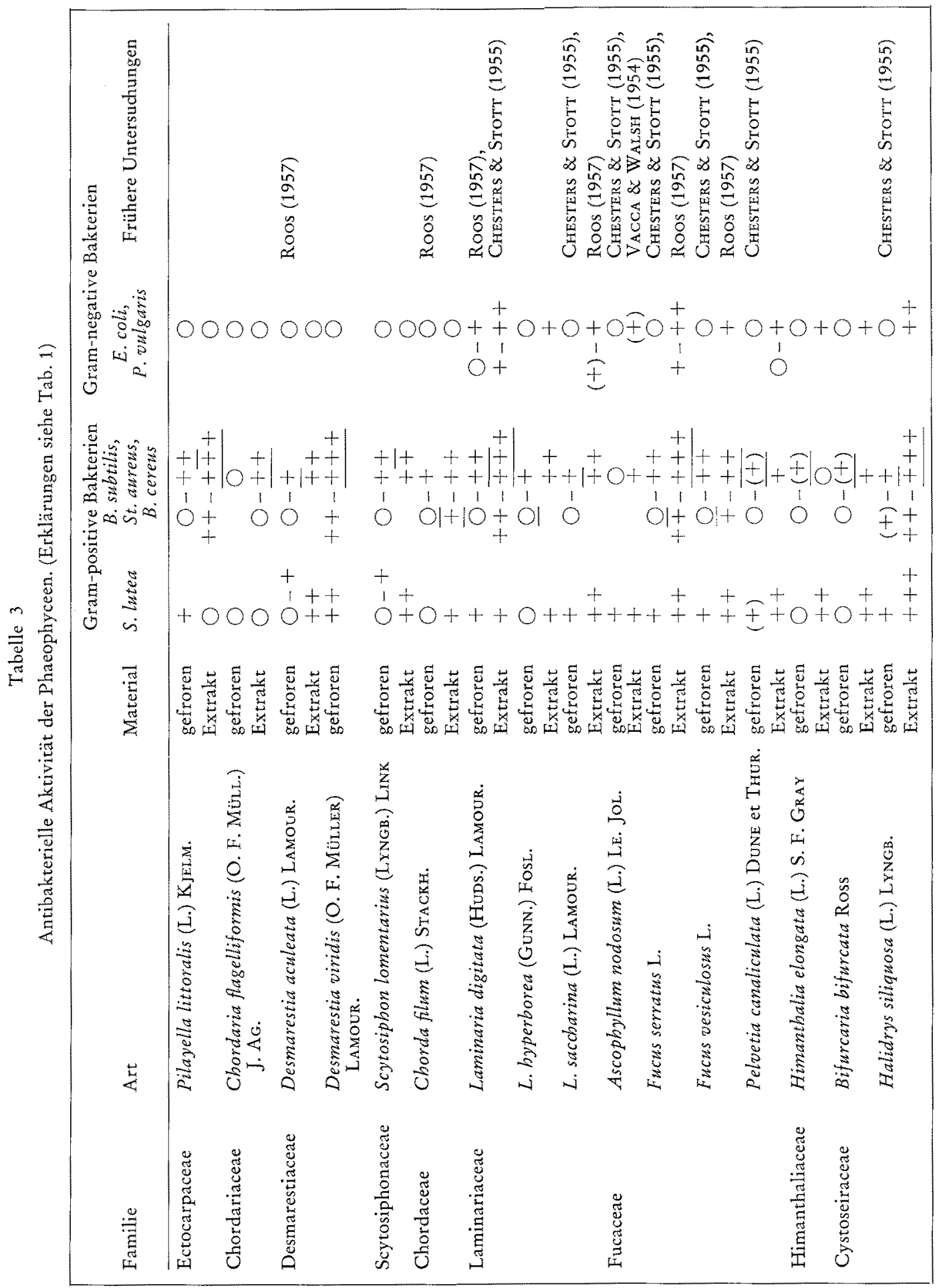


ten. Die Gattung Desmarestia ist ganz allgemein durch den Besitz von freier Schwefelsäure ausgezeichnet; diese wurde z. B. bei $D$. munda, $D$. latifrons und $D$. herbacea nachgewiesen (EPpley \& Bovel 1958). Neutralisiert man das Thalluspulver, so ist keine Hemmwirkung mehr vorhanden. Die antibakterielle Eigenschat von Desmarestia viridis ist also, wie schon SIEBURTH (1964) vermutete aber nicht experimentell bewiesen hat, lediglich auf eine $\mathrm{pH}$-Verschiebung des Kulturbodens zurückzuführen.

Halidrys siliquosa ist eine der wirksamsten Phaeophyceen. Das gefriergetrocknete, pulverisierte Material wurde zunächst nacheinander mit immer hydrophileren Lösungsmitteln extrahiert. Während z. B. bei den Chlorophyceen der mit Methanol extrainierbare Anteil den größten Teil der Aktivität enthielt, finden sich bei Halidrys größere Mengen antibakteriell wirksamer Substanzen auch in den lipophileren Fraktionen. Außerdem enthält aber auch der Extraktionsrückstand noch antibakterielle Stoffe, was auf hydrophilere nur mit Wasser extrahierbare oder hochmolekulare Substanzen schließen läßt. Ein ähnliches Bild ergibt sich, wenn man einen methanolischen Gesamtextrakt nacheinander mit hydrophoben und hydrophilen Lösungsmitteln behandelt.

Versuche zur säulenchromatographischen Trennung der am stärksten wirksamen äthanolischen Fraktion des Methanolgesamtextraktes an Kieselgel-Woelm mit der Lösungsmittelfolge Essigester/Aceton/Athanol/Methanol : Wasser $(7: 3)$ waren ebensowenig erfolgreich wie eine Trennung an Sephadex G 25 mit 50\%igem Alkohol. Es werden $z$ war einige aktive Fraktionen erhalten, die aber immer tiefbraun gefärbt waren. Außerdem lassen sich beide Säulen nicht mehr sauber auswaschen. Offensichtlich enthält Halidrys mehrere leicht zersetzliche Hemmstoffe.

\section{ZUSAMMENFASSUNG}

1. Die Aufarbeitungsmethoden zur Gewinnung antibakterieller Extrakte aus Meeresalgen werden im Hinblick auf Haltbarkeit und Trocknung des Materials untersucht.

2. Die antibakteriellen Eigenschaften der Präparate aus 9 Chlorophyceen, 16 Rhodophyceen und 16 Phaeophyceen gegen 4 gram-positive und 2 gram-negative Bakterienstämme werden bestimmt.

3. Die stärksten antibakteriellen Eigenschaften finden sich bei den Chlorophyceen. Von den Rhodophyceen haben nur Chondrus crispus, Laurencia pinnatifida, Delesseria sanguinea und zum Teil auch Rbodomela confervoides, von den Phaeophyceen Halidrys siliquosa, Fucus, Laminaria digitata und Desmarestia viridis eine deutliche Hemmwirkung.

4. Bei den Rhodophyceen und Chlorophyceen kommen überwiegend nur hydrophile, bei den Phaeophyceen auch mit lipophilen Lösungsmitteln extrahierbare Hemmstoffe vor.

5. Bei Dumontia incrassata liegen leicht flüchtige, schwach wirksame Hemmstoffe vor; bei Desmarestia viridis geht die Antibiose auf eine Verschiebung des $\mathrm{pH}$-Wertes des Kulturmediums durch die aus den Thalli hinausdiffundierende Schwefelsäure zurück. 
Danksagungen. Ich danke der Deutschen Forschungsgemeinschaft für die Gewährung von Sachmitteln und Herrn Prof. Dr. M. STEINer für das der Arbeit entgegengebrachte Interesse, den Mitarbeitern der Biologischen Anstalt Helgoland für ihre vielfältige Hilfe, Herrn Dr. Hartmann, Bonn, und M. Bert, Université de Caën, für ihre Hilfe bei der Bestimmung der Algen und Frl. A. WAGNer und Frl. S. RitTer für ihre Hilfe bei der Durchführung der Versuche.

\section{ZITIERTE LITERATUR}

Allen, M. B. \& DAwson, E. Y., 1960. Production of antibacterial substances by benthic tropical marine algae. J. Bact. 79, 459-460.

Chesters, C. G. C. \& Stott, J. A., 1955. The production of antibiotic substances by seaweeds. Int. Seaweed Symp. 2 (Trondbeim), 49-54.

Epplex, R. W. \& Bovell, C. R., 1958. Sulfuric acid in Desmarestia. Biol. Bull. mar. biol. Lab., Woods Hole 114, 101-106.

Gupta, A. B. \& Serrivastava, G. C., 1963. Production of an antibacterial substance by Hydrodictyon reticulatum. Curr. Sci. 32, 19-20.

HAAs, P., 1950. On certain peptides occurring in marine algae. Biochem. J. 46, 503-505.

Камтмото, K., 1955. Studies on the antibacterial substances extracted from seaweeds. 1, Effect of the extracts from seaweeds on the growth of some pathogenic organisms. Jap. J. Bact. $10,897-902$.

- 1956. Studies on the antibacterial substances extracted from seaweeds. 2. Effect of extracts from seaweeds on the growth of some acid fast bacteria. Jap. J. Bact. 11, 307-312.

McCutcheon, R. S., Arrigoni, L. \& Fischer, L., 1949. A phytochemical investigation of the kelps Cymathaere triplicata, Hedophyllum sessile and Egregia menziesii. J. Am. pharm. Ass. 38, 196-200.

Mautner, H. G., Gardner, G. M. \& Pratt, R., 1953. Antibiotic activity of seaweed extracts. 2. Rhodomela larix. J. Am. pharm. Ass. 42, 294-296.

Olesen, P. E., Maretzki, A. \& Almodovar, L. A., 1964. An investigation of antimicrobial substances from marine algae. Botanica mar. 6, 224-232.

Roos, H., 1957. Untersuchungen über das Vorkommen antimikrobieller Substancen in Meeresalgen. Kieler Meeresforsch. 13, 41-58.

Sieburth, J. McN., 1964. Antibacterial substances produced by marine algae. Devs. ind. Microbiol. 5, 124-134.

VACCA, D. D. \& $\mathrm{W}_{\mathrm{ALSH}}, \mathrm{R}$. A., 1954. The antibacterial activity of an extract obtained from Ascophyllum nodosum. J. Am. pharm. Ass. (scient. Ed.) 43, 24-26.

WeLCh, A. M., 1962. Preliminary studies of fungistatic properties of marine algae. J. Bact. 83, 97-99.

Anschrift des Autors: Dozent Dr. K.-W. Glombitza

Pharmakognostisches Institut

der Universität Bonn

53 Bonn

Nußallee 6 